\title{
Efficient Broadcasting with Guaranteed Coverage in Mobile Ad Hoc Networks
}

\author{
Jie Wu, Senior Member, IEEE, and Fei Dai, Student Member, IEEE
}

\begin{abstract}
We study an efficient broadcast scheme in mobile ad hoc networks (MANETs). The objective is to determine a small set of forward nodes to ensure full coverage. We first study several methods that guarantee coverage when the local view of each node on its neighborhood information is updated in a timely manner. Then we consider a general case where nodes move even during the broadcast process, making it impractical to maintain up-to-date and consistent local views. A formal framework is used to model inaccurate local views in MANETs, where full coverage is guaranteed if three sufficient conditions, connectivity, link availability, and consistency, are met. Three solutions are proposed to satisfy those conditions. First, we give a minimal transmission range that maintains the connectivity of the virtual network constructed from local views. Then, we use two transmission ranges, one for neighborhood information collection and the other for actual data transmission, to form a buffer zone that guarantees the availability of logical links in the physical network. Finally, we propose a mechanism called aggregated local view to ensure consistent local views. By these, we extend Wu and Dai's coverage condition for broadcasting in a network with mobile nodes. The effectiveness of the proposed scheme is confirmed via both performance analysis and simulation study.
\end{abstract}

Index Terms-Broadcasting, localized algorithms, mobile ad hoc networks (MANETs), mobility, simulation.

\section{INTRODUCTION}

B ROADCASTING a packet to the entire network is a basic operation and has extensive applications in mobile ad hoc networks (MANETs). For example, broadcasting is used in the route discovery process in several routing protocols, when advising an error message to erase invalid routes from the routing table, or as an efficient mechanism for reliable multicast in a fast-moving MANET. In MANETs with the promiscuous receiving mode, the traditional blind flooding incurs significant redundancy, collision, and contention, which is known as the broadcast storm problem [23]. Efficient broadcasting in a MANET focuses on selecting a small forward node set while ensuring broadcast coverage.

Broadcast protocols can be classified into deterministic and probabilistic approaches. The probabilistic approach [11], [23] usually offers a simple solution in which each node, upon receiving a broadcast packet, forwards the broadcast message with probability $p$. However, the probabilistic approach cannot guarantee full coverage. The deterministic approach guarantees full coverage and can be further classified based on the type of neighborhood information used: location-information-based and neighborset-based. In location-information-based broadcast protocols, location information of neighbors is available, whereas in neighbor-set-based broadcast protocols, only neighbor set information is available. Location information facilitates efficient broadcasting in terms of generating a small forward node set; however, it comes with a cost-location

- The authors are with the Department of Computer Science and Engineering, Florida Atlantic University, 777 Glades Rd., Boca Raton, FL 33431.E-mail: jie@cse.fau.edu,fdai@fau.edu.

Manuscript received 16 June 2004; revised 25 Aug. 2004; accepted 4 Oct. 2004; published online 23 Mar. 2005.

For information on obtaining reprints of this article, please send e-mail to: tmc@computer.org, and reference IEEECS Log Number TMC-0199-0604. information requires additional hardware, such as GPS. Other types of information can also be used which fall in between the above two models: directional information, where messages arrive from a certain angle-of-arrival (AOA), and distance information based on the signal strength received. All these models assume some sort of special hardware. In addition, location/direction/distance information may not be accurate. In this paper, we limit our consideration to deterministic broadcast protocols that use neighbor set information only, which corresponds to the weakest assumption on neighborhood information used.

In a broadcast process, each node decides its forwarding status based on given neighborhood information (such information is constructed from the neighbor set of each node), and the corresponding broadcast protocol is called self-pruning. In Fig. 1, black (white) nodes are forward (nonforward) nodes. Each circle corresponds to a one-hop neighborhood. Any source node is a black node by default. Basically, forward nodes form a connected dominating set (CDS), where each node in the system is either in the set or the neighbor of a node in the set. That is, each white node has at least one black neighbor. However, most existing broadcast schemes assume either the underlying network topology is static or quasi-static during the broadcast process such that the neighborhood information can be updated in a timely manner. The results in [7] show that existing static network broadcast schemes perform poorly in terms of delivery ratio when nodes are mobile. There are two sources that cause the failure of message delivery:

Collision. The message intended for a destination collides with another message. In Fig. 1, if messages from nodes $w$ and $x$ collide at node $y$, node $y$ does not receive any message.

Mobile nodes. A former neighbor moves out of the transmission range of the current node (i.e., it is no 


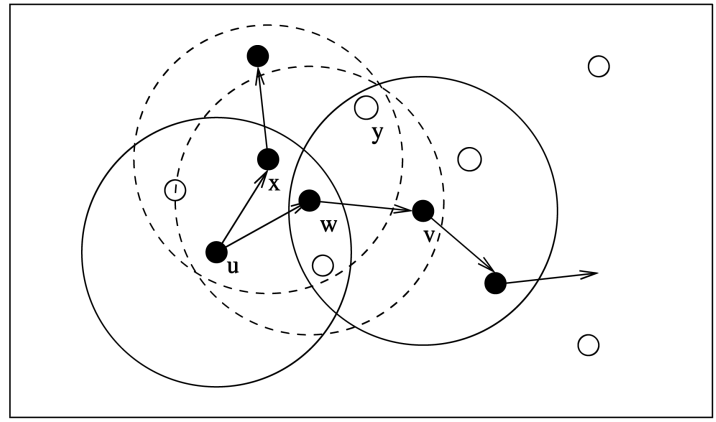

Fig. 1. Forward node set in a MANET.

longer a neighbor). In Fig. 1, when node $w$ moves out of the transmission range of $u$, the nodes along the branch rooted at $w$ of the broadcast tree will miss the message. ${ }^{1}$

Results in [7] show that the effect of collision can be relieved by a very short $(1 \mathrm{~ms})$ forward jitter delay, where a very high ( $\geq 99$ percent) delivery ratio is achieved in static networks. The majority of delivery failures are caused by mobile nodes. Therefore, in this paper, we focus on delivery failures caused by mobility only. The major challenges in designing a localized broadcast protocol while ensuring broadcast coverage are as follows: 1) The network topology changes over time, even during the broadcast process. 2) The local (1-hop) information is constructed based on "Hello" intervals. To avoid serious collision among "Hello" messages, nodes start their intervals asynchronously, making it difficult to ensure consistent local/global views among nodes. 3) The collection process for $k$-hop information incurs delay which may not reflect the current network topology when there are mobile nodes, even for a small $k$ in localized solutions.

As a consequence, the virtual network constructed from local views of nodes may not be connected (connectivity issue), its links may not exist in the physical network (link availability issue), and the global view constructed from the collection of local views may not be consistent (consistency issue).

In this paper, we first give a sufficient condition for connectivity at the physical network to ensure the connectivity at the virtual network. We then propose a solution using two transmission ranges to address the link availability issue. The neighborhood information, as well as the forward node set, are determined based on a short transmission range, whereas the broadcast process is done on a long transmission range. The difference between these two ranges is based on the update frequency and the speed of node movement. The difference is also used as a new controllable parameter to balance broadcast redundancy and broadcast delivery ratio. Although many deterministic broadcast protocols have been proposed with different broadcast redundancies (and collated broadcast delivery ratios), each broadcast protocol has only its "fixed" broadcast redundancy (and broadcast delivery ratio). It is, in general, hard to control redundancy and delivery for a given broadcast protocol. Note that the forwarding probability in

1. Nodes in the branch may still receive the message if some adjacent nodes of the branch forward the message. probabilistic broadcasting [23] is also a controllable parameter. However, it is difficult to establish a direct connection between parameter selection and node mobility. Finally, we propose a new mechanism called aggregated local view to ensure consistency of the global view. The conservative approach aggregates past $k$ local views in a special way to eliminate potential view inconsistency caused by asynchronous "Hello" intervals and collection process delay for $k$-hop information at each node.

The main contributions of this paper are as follows:

1. Propose the first localized broadcast protocol that can handle node mobility while ensuring broadcast coverage.

2. Systematically address the issue of inconsistent local view caused by neighborhood information delay, asynchronous "Hello" intervals, and node mobility.

3. Introduce a new controllable parameter to balance broadcast efficiency and broadcast delivery ratio.

4. Conduct a simulation study to verify the effectiveness of the new approach.

The remainder of the paper is organized as follows: Section 2 provides some preliminaries, especially $\mathrm{Wu}$ and Dai's coverage condition, and motivations. Section 3 proposes the mobility control method based on two transmission ranges, presents the consistent global view construction through aggregation of local views, and gives some analytical study and optimization techniques. Simulation results are presented in Section 4 . The paper concludes in Section 5.

\section{Preliminaries and Motivations}

This section starts with some related work on mobility management and, in particular, neighbor set management in a mobile environment. Then, an overview of broadcast protocols in MANETs based on self-pruning is given. The focus is on $\mathrm{Wu}$ and Dai's coverage condition and six existing protocols as its special cases. Finally, we focus on the limitation of $\mathrm{Wu}$ and Dai's coverage condition in dynamic networks to motivate this study.

\subsection{Mobility Management}

The capacity of MANETs is constrained by the mutual interference of concurrent transmissions between nodes. The mobility of nodes adds another dimension of complexity in the mutual interference. Camp et al. [4] gave a comprehensive survey on mobility models for MANETs. Several studies [10] focused the effect of mobility on the network capacity. The impact of mobility on performance of routing protocols is discussed in [2].

Very little work has been done in maintaining accurate neighborhood information in a mobile environment without increasing the frequency of "Hello" messages. One exception is [12], where a stable zone and a caution zone of each node have been defined based on a node's position, speed, and direction information obtained from GPS. Specifically, stable zone is the area in which a mobile node can maintain a relatively stable link with its neighbor nodes since they are located close to each other. Caution zone is the area in which a node can maintain an unstable link with 
its neighbor nodes since they are relatively far from each other. The drawback of this approach is that it is GPS-based, which comes with a cost. In addition, there is no rigorous analysis on the impact of mobility on the selection of these two zones.

Several papers [3] address the time period that two nodes will remain close enough in proximity for a link between them to remain active. Several routing protocols, associativity-based routing (ABR) [22] and signal stability-based adaptive routing (SSA) [8], have been proposed that select stable links to construct a route. In [20], GPS information is used to estimate the expiration time of the link between two adjacent hosts. Recently, several studies have been done on the effect of mobility on routing path duration [17]. However, no broadcast protocol uses the notion of stable link to evaluate the stability of a neighbor set in order to better decide the forwarding status of each node. Although several probabilistic broadcast protocols [11], [23] have been proposed by trading between efficiency (simple design) and coverage (delivery ratio), it is difficult to establish a direct connection between forwarding probability and node mobility.

\subsection{Broadcast Protocols Based on Self-Pruning}

A MANET is usually modeled as an undirected graph $G=(V, E)$, where $V$ is a set of mobile nodes and $E$ is a set of wireless links. A link exists between two nodes $u$ and $v$ if and only if their physical distance is less than a transmission range $r$. Wu and Dai [24] proposed a generic efficient broadcast protocol based on self-pruning. In a self-pruning protocol, each node determines its forwarding status based on its local $k$-hop information, where $k=2$ or 3 . Every node is a forward node by default and becomes a nonforward node (pruned) when a sufficient coverage condition holds, as will be discussed later. For a node $v \in V$, its exact $k$-hop neighbor set, $H_{k}(v)$, is the set of nodes that is exactly $k$-hops away from $v$, and its $k$-hop neighbor set, $N_{k}(v)=\{v\} \cup H_{1}(v) \cup H_{2}(v) \cup \ldots \cup H_{k}(v)$, is the set of nodes that is at most $k$ hops away from $v$. The $k$-hop information of $v, G_{k}(v)$, is the induced graph of $N_{k}(v)$, excluding links among nodes in $H_{k}(v)$. For example, links between two nodes exactly 2 hops away are included in 3-hop information, but not in 2-hop information. Each node builds its $k$-hop information by exchanging $(k-$ 1)-hop information with its neighbors via periodical "Hello" messages. Therefore, $k$ rounds of exchanges of the accumulative neighbor set between neighbors are needed to collect $k$-hop information at each node.

The "Hello" messages also propagate the priority of each node, which could be a static property (e.g., node id) or a dynamic one (e.g., node degree). During a broadcast process, each node may also extract from the incoming broadcast packets a list of visited nodes that have forwarded the broadcast packet. Using $k$-hop information, priority, and visited node information, each node decides its own status, forwarding/nonforwarding, based on the following coverage condition:

Coverage Condition [24]. Node $v$ has a nonforward node status if for any two neighbors $u$ and $w$, a replacement path exists that connects $u$ and $w$ via several intermediate nodes (if any)

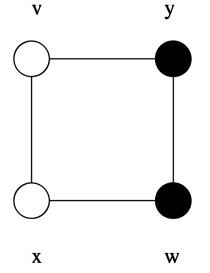

(a)

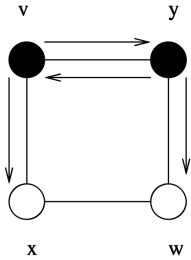

(b)
Fig. 2. (a) Forward node set without history information (static). (b) Forward node set with upstream history information (dynamic) with node $v$ being the source (visited node).

with either higher priority values than the priority value of $v$ or with visited node status.

Assume node id is used as priority. Node $x$ in Fig. 2a is a nonforward node based on the coverage condition because its neighbors, $v$ and $w$, are connected via a replacement path that contains only intermediate nodes (in this case, $y$ ) with higher node id than $x$. Node $y$ is a forward node because no such replacement path exists. It was proved in [24] that the coverage condition ensures the coverage; that is, the forward nodes, including the source, form a connected dominating set (CDS) of $G$, if $G$ is connected. Therefore, the broadcast packet is delivered to all nodes in $V$ if no packet is lost due to node mobility or MAC layer collision. Six existing algorithms were shown in [24] to be special cases of the coverage condition. They are $\mathrm{Wu}$ and $\mathrm{Li}^{\prime} \mathrm{s}$ marking process with Rules 1 and 2 [26], Dai and Wu's Rule $k$ [6], Chen et al.'s Span [5], Sucec and Marsic's LENWB) [21], Peng and Lu's SBA [15], and Stojmenovic's algorithm [19]. Fig. 3 shows a sample broadcasting based on self-pruning.

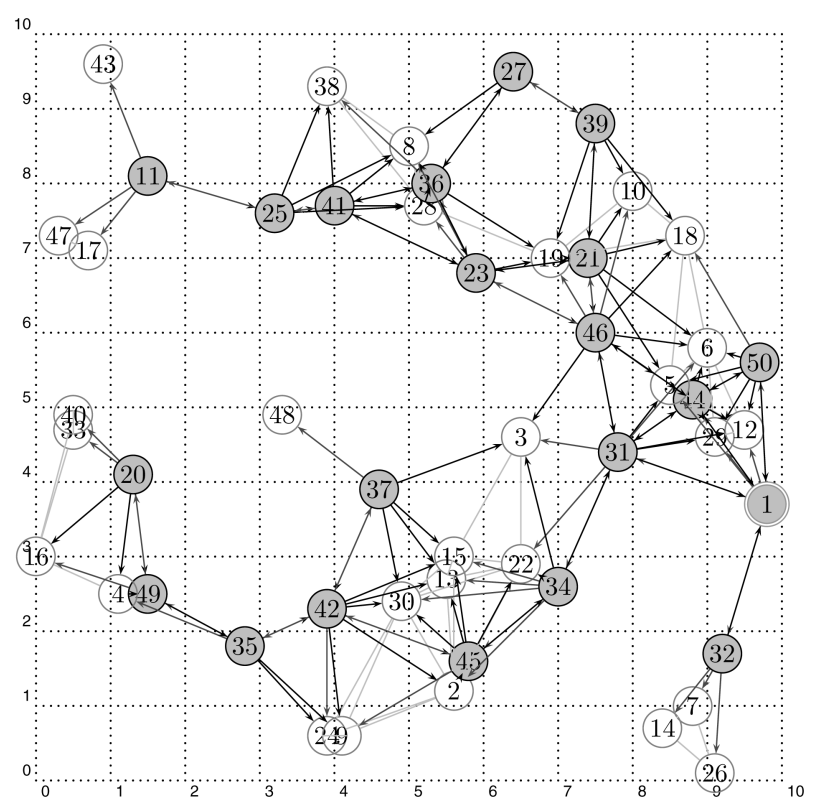

Fig. 3. A sample broadcast process. Gray nodes are forward nodes and white nodes are nonforward nodes. Arrows represent receptions of the broadcast packet. 
A self-pruning protocol is static if it does not use visited node information in the coverage condition; otherwise, it is dynamic. In a static protocol, forward nodes are predetermined before any broadcast process and are source independent. For example, node $w$ in Fig. 2a is a forward node in a static protocol because there is no replacement path to connect neighbors $x$ and $y$. When node $v$ is the source, there are actually three forward nodes $v, w$, and $y$. In a dynamic protocol, the forward status is determined during the broadcast process. Each node usually determines its status after a short backoff delay when the node receives the packet for the first time. During the backoff delay, more neighbors may forward the same broadcast packet and those neighbors become visited nodes. Each broadcast packet carries a list of recently visited nodes and each node uses the visited node information to enhance the chance of being pruned. The number of forward nodes is usually smaller than in a static protocol, but the selection of forward nodes is usually source dependent. Fig. $2 b$ shows the broadcast process in the same network and with the same source node as in Fig. 2a. This time, node $w$ is a nonforward node because nodes $x$ and $y$ are connected via node $v$, which is known by $w$ as a visited node (such information is piggybacked with the broadcast packet). There are only two forward nodes $v$ and $y$.

In [24], it is assumed that local views of the broadcast specific information (i.e., visited node information) are dynamic but safe, i.e., an unvisited node will not be mislabeled as visited and those of the broadcast independent information (i.e., $k$-hop information and priority) are "static" and accurate during a broadcast process. However, in mobile networks, such "static" information usually changes and causes inaccurate local views. Based on these inaccurate views, full coverage (i.e., 100 percent delivery ratio) is not guaranteed. Recently, we conducted a simulation study on the performance of the coverage condition and its special cases [7]. Simulation results show that most efficient broadcast protocols suffer from a low delivery ratio in highly mobile networks.

\section{Proposed Method}

This section proposes a mobility control method that addresses connectivity, link availability, and consistency issues. Three sufficient conditions are given: the first one on the connectivity of the physical network to ensure connectivity of the virtual network, the second one on the bound of the range difference to ensure link availability, and the third one on the consistent local views to ensure correct decision made at each node. Two mechanisms, called buffer zone and aggregated local view, are proposed to satisfy the latter two conditions. Finally, we introduce methods to relax these stringent sufficient conditions based on probabilistic analysis and optimization techniques.

\subsection{Logical Network and Broadcast State}

In [24], the coverage condition was applied on a static or quasi-static physical network. In a quasi-static physical network, the physical topology stops to change several "Hello" intervals before a broadcast process and stays

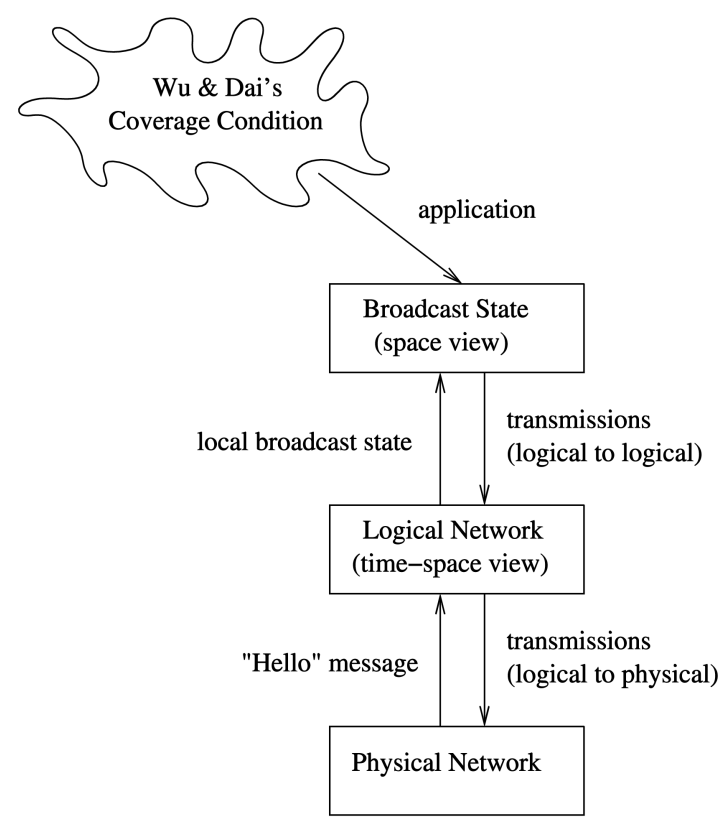

Fig. 4. The mapping from the logical network and broadcast state to the physical network.

unchanged until the broadcast process completes. For the sake of clarity, we assume node id is used as priority.

Two Levels of Abstraction. The correctness of the coverage condition is based on the assumption that the local view is an accurate and immediate reflection of the physical topology. In MANETs, however, this assumption can be easily invalidated due to the continuous node mobility. In fact, in order to apply the coverage condition on MANETs with potentially outdated local views, we introduce the concepts of logical network, a dynamic virtual network constructed from all local views, broadcast state, a snapshot of local views during a broadcast process, and two levels of abstraction, as shown in Fig. 4:

1. Level-1 abstraction: from physical network (timespace view) to logical network (time-space view).

2. Level-2 abstraction: from logical network (timespace view) to broadcast state (space view).

A logical network is the collection of all local views, i.e., a supergraph containing all the nodes and links in local views.

Definition 1. The local view, $G_{k}^{\prime}(v, t)=\left(N_{k}^{\prime}(v, t), E_{k}^{\prime}(v, t)\right)$, of node $v$ is its $k$-hop information collected at time $t$. The logical network, $G^{\prime}(t)=\left(V, E^{\prime}(t)\right)$, is the union of all local views at time $t$, where $E^{\prime}(t)=\bigcup_{v \in V} E_{k}^{\prime}(v, t)$.

Both local view and logical network are time sensitive. When the physical topology changes, the change is detected by "Hello" messages and reflected in the logical network. Consider the MANET in Fig. 5a. We assume that each node has the same "Hello" interval $f_{,}^{2}$ but each node starts its period asynchronously. Fig. $5 \mathrm{~b}$ shows the update of local views. We label the time each node sends its last "Hello"

2. The condition can also be be relaxed in a controllable way, such as $(1 \pm 0.25) f$ in AODV. 


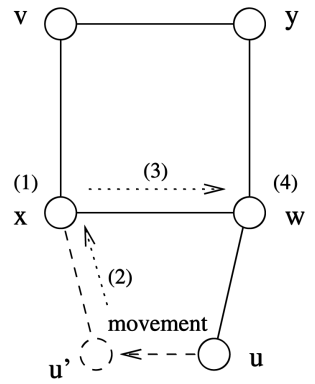

(a)

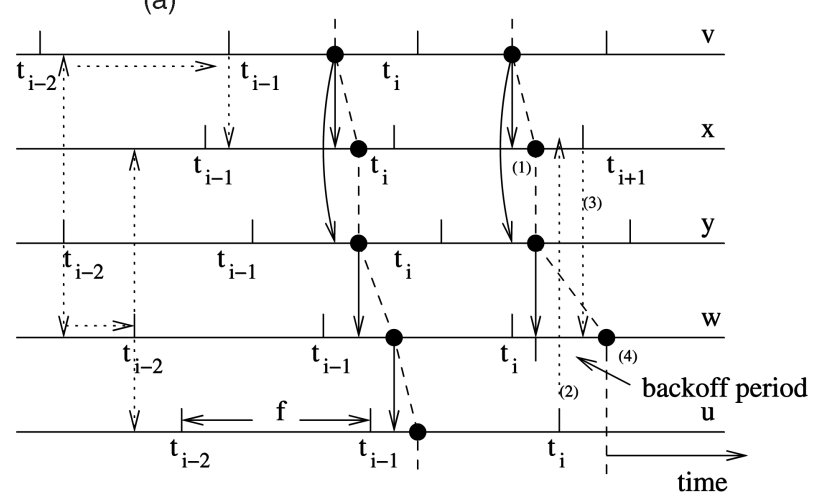

(b)

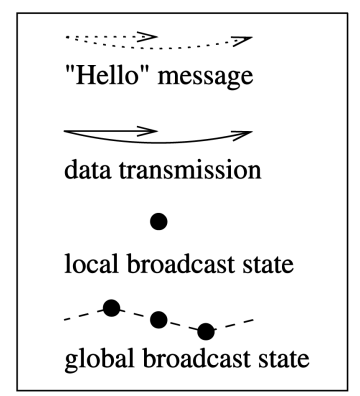

time
Fig. 5. (a) A physical network and (b) the corresponding time-space view during two broadcast processes. The first broadcast process starts after time $t_{i-1}$ and succeeds. The second one starts after time $t_{i}$ and fails due to inconsistent local views.

message before the broadcasting as $t_{i}$, the time for previous "Hello" messages as $t_{i-1}, t_{i-2}$, and so on. Note that $t_{i}$ at each node may refer to different physical time. Here each node builds 2-hop information. If node $y^{\prime}$ "Hello" message is first received by node $v$ between $t_{i-2}$ and $t_{i-1}$ (the "Hello" message propagation is shown in a dotted arrow line), it is added to $v / \mathrm{s} 1$-hop neighbor set, which is advertised in $v^{\prime} \mathrm{s}$ next "Hello" message at $t_{i-1}$. That is, link $(v, y)$ is added to local views of nodes $v$ and $x$. Similarly, link $(w, y)$ is also detected and added to local views of nodes $u, w$, and $x$.

Broadcast state is a snapshot of local views of the logical network. For a specific broadcast process, broadcast state forms a virtual static network, upon which the coverage condition is applied.

Definition 2. A local broadcast state $G_{k}^{\prime \prime}(v)=\left(N_{k}^{\prime}\left(v, t_{v}\right)\right.$, $\left.E_{k}^{\prime}\left(v, t_{v}\right)\right)$ of node $v$ for a broadcast is its local view at the time $t_{v}$, when it makes its forwarding/nonforwarding decision. A global state $G^{\prime \prime}=\left(V, E^{\prime \prime}\right)$, is the union of all local broadcast states, where $E^{\prime \prime}=\bigcup_{v \in V} E_{k}^{\prime}\left(v, t_{v}\right)$.

In Fig. 5b, the time that each individual node makes its decision is marked with a black dot. Note that local broadcast states are taken at the times marked by these black dots and the global broadcast state is the collection of local broadcast states (marked by the dashed line connecting all black dots). Suppose node $v$ in Fig. 5a issues a broadcasting, where nodes $y$ and $w$ become forward nodes. The corresponding broadcast state is shown in Fig. 5b. The source node $v$ samples its local state at the beginning of the broadcasting, between $t_{i-1}$ and $t_{i}$. On receiving the broad- cast packet from $v$, both nodes $x$ and $y$ wait for a random backoff delay and determine their forwarding status. Node $x$ becomes a nonforward node and node $y$ becomes a forward node. Nevertheless, both nodes sample their local state at the time the decision is made. Subsequently, nodes $w$ and $u$ receive the broadcast packet and make their decision based on their local state. The global state is the union of all local states, which are sampled at different physical times during the broadcast process.

Next, we examine the "gap" at each level of abstraction. The gap is caused by various synchronization delays and protocol handshakes at each level of abstraction. We will look at potential problems caused by the gap and, in the next section, we will present solutions to these problems.

Gap in level 1 abstraction. In order to build $k$-hop information, each node advertises its $(k-1)$-hop information via "Hello" messages. Each node updates its local view based on received "Hello" messages. Because of asynchronous periodic exchanges among neighboring nodes, the 1-hop neighbor set in a local view at a particular time $t$ does not reflect the actual neighbor set at time $t$, but the offset is bounded by the "Hello" interval $f$. In fact, $k$-hop information is a set that consists of neighborhood information sampled at different times. In general, $H_{i+1}(u)$ was sampled one interval after $H_{i}(u)$ for $i=1,2, \ldots, k-1$. Clearly, the $k$-hop information at time $t$ does not reflect the actual neighborhood topology at time $t$ and the offset is bounded by $k f$. Suppose the speed of node movement is upper bounded by $s$. Then, $s f$ is the maximum distance a node can move around during a "Hello" interval. The maximum relative distance between two nodes in such an interval is $\Delta=2 s f$.

Gap in level 2 abstraction. In a broadcast process based on self-pruning, each node follows three steps: 1) first receipt of broadcast message, 2) backoff delay, and 3) forward/nonforward status decision and transmission (if needed). A broadcast period starts from the source sending out the message and ends with the last node deciding its forwarding status. Like [24], it is assumed that the broadcast message propagates quickly and its delay can be ignored. Backoff at intermediate nodes is allowed, but accumulative backoff along each path of the broadcast tree is bounded by $b$, called broadcast delay, for each broadcast. Note that $b$ may also include broadcast message propagation delay if such delay cannot be neglected.

\subsection{Mobility-Sensitive Broadcasting}

$\mathrm{Wu}$ and Dai's coverage conditions can be applied to the global broadcast state and ensures coverage, given that the following three conditions are met: connectivity, link availability, and consistency. The first two conditions resolve the gaps at level 1 and level 2 abstractions, respectively. The third condition ensures the consistent use of local views.

Connectivity. The virtual network that corresponds to the global broadcast state should be connected in order to apply $\mathrm{Wu}$ and Dai's coverage condition. The following theorem shows the density requirement at the physical network for ensuring a connected virtual network:

Theorem 1. If the physical network with transmission range $r_{1}-\Delta^{\prime}$ is connected under all time, where $\Delta^{\prime}=2 s(f+b)$, 


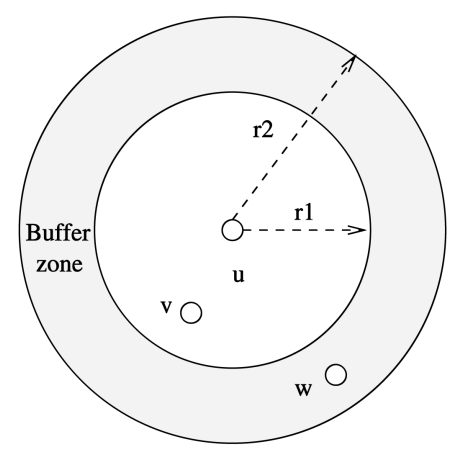

Fig. 6. Forward node selection and forwarding process based on two different transmission ranges: $r_{1}$ and $r_{2}$.

then every virtual network induced from a global broadcast state is connected.

Proof. Assume the global broadcast state is taken in a broadcast process started at time $t$. Since the maximum broadcast delay is $b$, all local states are taken within time period $[t, t+b]$. If the distance of two nodes $u$ and $v$, $d(u, v) \leq r_{1}-2 s(f+b)$ at time $t-f$, then $d(u, v) \leq r_{1}$ during $[t-f, t+b]$. Suppose $u$ takes its local broadcast state at $t_{u} \in[t, t+b]$, it must have received $v^{\prime}$ s last "Hello" message in $\left[t-f, t_{u}\right]$. Therefore, link $(u, v)$ exists in $u^{\prime}$ s local broadcast state. Since the global broadcast state consists of all links from local broadcast state and the network is connected at time $t-f$ in the range of $r_{1}-\Delta^{\prime}$, the corresponding virtual network induced from the global broadcast state is also connected.

Theorem 1 poses a rather strict connectivity requirement on the physical network. That is, if the physical network cannot meet the connectivity requirement, the virtual network is not guaranteed to be connected and $\mathrm{Wu}$ and Dai's approach will fail. We will discuss later an approach that relaxes the connectivity requirement under the cost of pruning efficiency.

Link availability. Any link in the global broadcast state should still exist in the physical network during the broadcast period. We propose to use two transmission ranges, $r_{1}$ and $r_{2}$, with $r_{1}<r_{2} . r_{1}$ is used to collect neighbor set and $k$-hop information through "Hello" messages, whereas $r_{2}$ is used to perform actual transmission. A node that is within the range of $r_{1}$ of node $u$ is called a neighbor of $u$ and the collection of such nodes is the neighbor set of $u$. The set of nodes that are reachable based on $r_{2}$ is called effective neighbor set. Fig. 6 shows the relationship between these two transmission ranges. In this example, $v$ is in $u^{\prime} \mathrm{s}$ neighbor set (also in $u^{\prime}$ s effective neighbor set), whereas $w$ is in $u^{\prime}$ s effective neighbor set (but not in $u^{\prime}$ s neighbor set).

Theorem 2. To ensure the link availability requirement, $r_{2}$ should be set so that $\Delta^{\prime \prime} \leq r_{2}-r_{1}$, where $\Delta^{\prime \prime}=k \Delta+\Delta^{\prime}$ and $k$ for $k$-hop information.

Proof. (sketch) We need to show that any neighbor under the transmission range $r_{1}$ when its state is sampled is still an effective neighbor under the transmission range $r_{2}$ when the message is sent out. The total delay includes $k$-hop neighbor set collection that takes $k$ intervals and $(f+b)$ for broadcast and synchronization delay. The

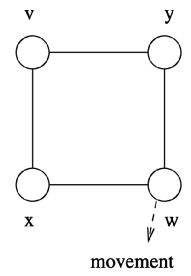

(a)

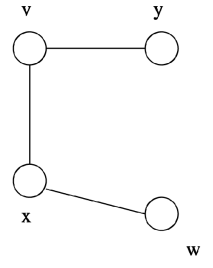

(b)

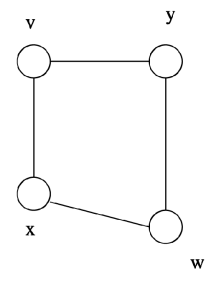

(c)
Fig. 7. The physical network (a) before and (b) after the movement, and (c) the aggregated local view of node $y$.

former contributes a distance of $k \Delta$ and the latter $\Delta^{\prime}$.

The above theorem provides some theoretical foundations for ensuring full coverage. However, the analysis shows only the worst case situation, which rarely occurs. Later we will show that even when $r_{2}-r_{1}$ is much smaller than $\Delta^{\prime \prime}$, the probability of an undetected link failure is very low. Since most self-pruning protocols have certain degrees of redundancy, it usually takes several link failures to cause a global delivery failure. Therefore, the probability can still be high that a high delivery ratio can be achieved with a relatively small buffer zone width. There is a wide range of potential tradeoffs between broadcast efficiency and broadcast delivery ratio.

The idea of two transmission ranges is to use the "ring," the area bounded by two circles with transmission ranges $r_{1}$ and $r_{2}$, as a buffer zone to nullify the various bad effects caused by node mobility and transmission delay. However, one bad effect, called inconsistent local views, cannot be nullified no matter how wide the buffer zone is. Inconsistent local views ultimately result in a bad decision from a node. A decision is bad if a node that should forward the message decides on a nonforwarding status.

Consistency. Two local views (or states for a specific broadcasting) of nodes $u$ and $v$ are inconsistent, if a link $(v, w)$ exists in $u^{\prime}$ s local view (state), but $v$ does not view $w$ as a 1-hop neighbor. For example, assume the physical topology in Fig. 2 changes shortly before the broadcast. The broadcast may fail due to inconsistent views. Fig. 7a shows the physical network before the change, where node $x$ is a nonforward node because its neighbors $v$ and $w$ are connected via a replacement path $(v, y, w)$. Fig. $7 \mathrm{~b}$ shows the physical network before the broadcast, where $y$ is a nonforward node because $w$ is no longer a neighbor. Node $y$ detects the broken link $(y, w)$ before node $x$, since $y$ is adjacent to the link, whereas $x$ is 2-hop away from the link. Both nodes may take a nonforwarding status in the broadcast, $x^{\prime}$ s decision based on the outdated view and $y^{\prime} \mathrm{s}$ based on the updated view. Therefore, node $w$ may never receive the broadcast packet.

We say node $v^{\prime}$ s local view (state) is consistent with the logical network (global state) (or simply consistent), if its local view (state) contains all its adjacent links in the logical network (global state). Formally speaking, let $H_{1}(v)$ denote $v$ 's exact 1-hop neighbor set in its local view (state), and $H_{1}^{*}(v)$ its exact 1-hop neighbor set in the logical network (global state). The local view (state) is consistent if $H_{1}(v)=H_{1}^{*}(v)$. The following lemma and theorem show 


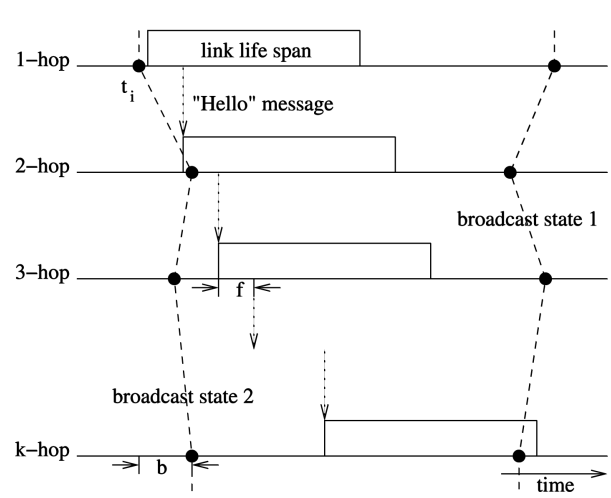

(a)

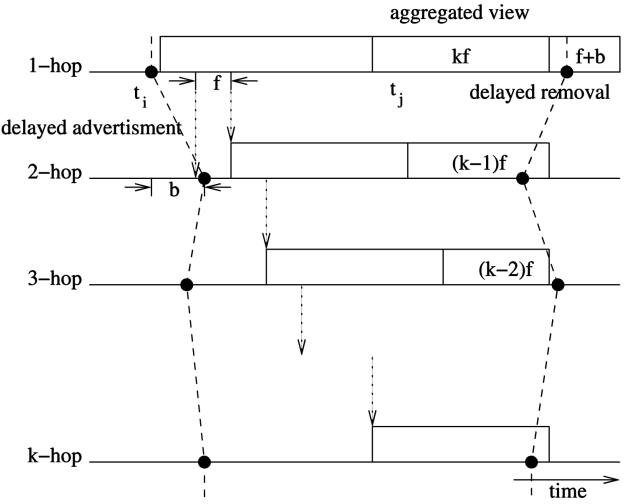

(b)

Fig. 8. Life spans of a single link in local views of neighboring nodes. (a) Inconsistent local states may be sampled due to inconsistent local views and/or the broadcast delay. (b) Local states sampled from aggregation local views enhanced by delayed advertisement and delayed removal.

that this definition of consistency, together with the connectivity and link availability conditions, are sufficient conditions to ensure full delivery in a self-pruning protocol.

Lemma 1. If the global state $G^{\prime \prime}$ is connected and local states $G^{\prime \prime}(v)$ of all nodes $v$ are consistent, then the forward node set determined by applying $W u$ and Dai's coverage condition on each node's local states form a CDS of $G^{\prime \prime}$.

Proof. Suppose $F$ is the forward node set derived using $\mathrm{Wu}$ and Dai's coverage condition on $G^{\prime \prime}, F$ is a CDS of $G^{\prime \prime}$ (connectivity condition). Let $F^{\prime}$ be the forward node set derived using $\mathrm{Wu}$ and Dai's condition on local state $G^{\prime \prime}(v)$ of each node $v$. Consider each nonforward node $v \notin F^{\prime}$. In $v^{\prime}$ s local state, any two neighbors in $H_{1}(v)$ are connected via a replacement path in $G^{\prime \prime}(v)$. Since $G^{\prime \prime}(v)$ is a subgraph of $G^{\prime \prime}$ and $H_{1}(v)=H_{1}^{*}(v)$ (consistency condition), any two neighbors in $H_{1}^{*}(v)$ are also connected by a replacement path in $G^{\prime \prime}$. Therefore, $v \notin F$. That is, $F^{\prime}$ is a superset of $F$ and a CDS of $G^{\prime \prime}$.

Theorem 3. If the connectivity, link availability, and consistency conditions are all satisfied, then $W u$ and Dai's generic selfpruning protocol ensures full coverage.

Proof. Based on the connectivity and consistency conditions, the global state is connected and consistent. From Lemma 1, the corresponding forward node set $F^{\prime}$ is a CDS of the global broadcast state. Based on the link availability condition, each link in the global broadcast state corresponds to a valid link in the physical network. Therefore, all nodes in the physical network finally receive the broadcast.

Next, we propose to use the aggregated local view to address the inconsistency problem. The inconsistency in the above example occurs when node $y$ removes link $(y, w)$ in its local view before node $x$ does so. As shown by the broadcast state 1 in Fig. 8a, a broken link is first detected by the end nodes (1-hop neighbors). This link is not removed from local views of other nodes until the link failure is advertised via "Hello" messages. When $k$-hop information is used, it takes up to $k$ "Hello" intervals for all affected nodes to update their local views. The solution is that once $a$ node advertises its 1-hop neighbor set, it cannot back away from it immediately. Each node $v$ keeps $k$ recent versions of its local view and uses the aggregation of those local views to make the forwarding/nonforwarding decision.

More formally, let $G_{k}^{0}(v)=\left(N_{k}(v), E_{k}(v)\right)$ be the current local view of $v$ and $G_{k}^{-i}(v)$ be the local view that is $i$ "Hello" intervals before the current one (or simply called view $-i$ ). Let $L_{j}(v)=\left(H_{j-1}(v) \times H_{j}(v)\right) \cap E_{k}(v)$ contain links between $v^{\prime}$ s $(j-1)$-hop and $j$-hop neighbors. Links in $L_{j}(v)$ have an "age" of $j$; i.e., this information takes $j$ rounds of "Hello" exchanges to build up. Also, $L_{j}^{-i}(v)$ corresponds to the $L_{j}(v)$ in view $-i$, which has an age of $i+j$. The aggregated local view of $v, G_{k}^{\prime}(v)$, is defined as follows:

$$
G_{k}^{\prime}(v)=\left(N_{k}(v), L_{1}^{\prime}(v) \cup L_{2}^{\prime}(v) \cup \ldots \cup L_{k}^{\prime}(v)\right),
$$

where

$$
L_{j}^{\prime}(v)=L_{j}(v) \cup L_{j}^{-1}(v) \cup \ldots \cup L_{j}^{-(k-j)}(v)
$$

for $1 \leq j \leq k$. Note that the maximal age of $L_{j}^{\prime}(v)$ is $k$.

In aggregated local views, the life span of each link is extended to compensate for the propagation delay of the link failure information. Aggregated local views are consistent; that is, local broadcast states sampled at the same physical time would be consistent (e.g., when the broadcast process completes instantly). Fig. 7c shows the aggregated local view of node $y$. Based on this local view, node $y$ will still forward. Intuitively, once a node $v$ appears as a neighbor of $u$ (in the range of $r_{1}$ ) during the recent $k$ intervals, it still has to be treated as a neighbor even if it currently moves out of $u^{\prime}$ s visible range but is still in $u^{\prime}$ s effective neighbor set (as shown in Theorem 2).

Another cause of inconsistency is the backoff delay, where a new link is detected during a broadcast process. As shown in Fig. 5, node $u$ is initially a neighbor of $w$ and later moves to $x$ as its neighbor. If a "Hello" message is sent from $x$ to $w$ after $x$ has made its decision, but before $w^{\prime}$ s decision is made, then $w^{\prime}$ s decision is made based upon information that is not available to $x$. Consider the following sequence of events as shown in Fig. 5:

1. $x$ decides its nonforwarding status,

2. $u$ is detected by $x$ as a new neighbor,

3. $x$ advertises its new neighbor set, and 


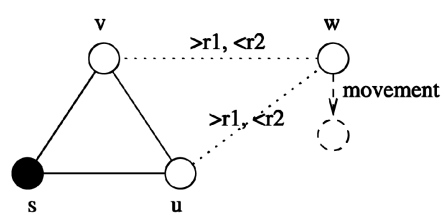

(a)

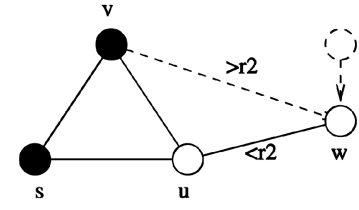

(b)
Fig. 9. A network with one mobile node $w$ (a) before the movement and (b) after the movement. Dotted lines represent undetected physical links. The dashed line represents an undetected broken link. (a) "Hello" range $r 1$ causes partition. (b) "Hello" range $r 2$ causes link failure.

4. $w$ believes that $u$ is covered by $x$ and becomes a nonforward node.

In this case, $u$ will never receive the broadcast packet. This situation is illustrated in Fig. 8a as broadcast state 2, where life spans of a single link in local views of neighboring nodes $(1,2, \ldots, k$ hops away from the link) are shown. The new link is detected after a 1-hop neighbor has made its decision, and is propagated to a 2-hop neighbor before a decision is made there. One solution is the delayed advertisement. As shown in Fig. 8b, a 1-hop neighbor will hold the advertisement of a new link if its age is younger than $b$, the maximal time period of a broadcast process. Thus a new link detected during a broadcast process will not affect the decision of other nodes.

Two other problems may cause inconsistency in aggregated local views. The first one is illustrated by the broadcast state 1 in Fig. 8a. When the broadcast process begins at a $k$-hop neighbor and ends at a 1-hop neighbor of a broken link, this link may be removed from the 1-hop neighbor's local view before the local state is sampled. Another problem is that a link failure may be detected by two end nodes in different times, a time skew bounded by the "Hello" interval $f$. Both problems can be solved by the delayed removal of a link from local views of its end nodes with a delay of $f+b$. Fig. 8b shows the effect of delayed advertisement, aggregated view, and delayed removal. After using delayed advertisement, if a link does not appear in the local view of a 1-hop neighbor, it will not appear in a $i$-hop $(i \geq 2)$ neighbor's local state either. After using the aggregated view and delayed removal, the removal of a link from the local views of its 1-hop neighbors is extended by $k f+f+b$. Because this link will be removed from local views of all $i$-hop neighbors within $k$ "Hello" intervals, the local views are always consistent. In addition, the time skew between two 1-hop nodes and the broadcast delay are compensated by the additional delay of $f+b$. Therefore, if a link appears in the local state of an $i$-hop neighbor, it will also appear in both 1-hop neighbors' local views. The following theorem can be easily proven based on the above reasoning:

Theorem 4. If each node maintains its aggregated local view with delayed advertisement and delayed removal, then all local broadcast states sampled from these local views are consistent.

\subsection{Implementation Details}

According to Theorem 1, full coverage is guaranteed only when the network is sufficiently dense (i.e., connected with transmission range $r_{1}-\Delta^{\prime}$ ). In the following, we propose a mechanism, called dual neighbor sets, to relax the connectivity requirement under the cost of pruning efficiency. In sparse networks, using a small "Hello" transmission range may cause partition in the logical network. As shown in Fig. 9a, when the "Hello" transmission range is $r_{1}=r_{2}-\Delta^{\prime}$, neither $u$ nor $v$ views $w$ as a neighbor because they cannot receive "Hello" messages from node $w$. Therefore, both nodes $u$ and $v$ are nonforward nodes and no one will forward the broadcast packet to node $w$. On the other hand, using a larger $r_{1}$ violates the link availability condition in Theorem 2 . The broadcast process may fail due to the lack of a buffer zone. Fig. $9 \mathrm{~b}$ shows the situation when $r_{1}=r_{2}$ is used. Node $u$ is a nonforward node and node $v$ is supposed to forward the broadcast packet to node $w$. However, node $w$ moves out of the transmission range of $v$ and never receives this packet.

Our solution is based on maintaining two neighbor sets. The effective neighbor set consists of all nodes within the actual transmission $r_{2}$ and the advertised neighbor set consists of only nodes with distance less than $r_{1}$. If $v$ is a nonforward node, every pair of nodes in $v^{\prime}$ s effective neighbor set must be connected via a replacement path. In this case, node $v$ in Fig. 9a views $w$ as a neighbor and becomes a forward node. On the other hand, only the advertised neighbor set is propagated to its $k$-hop neighbors. Because link $(v, w)$ in Fig. $9 \mathrm{~b}$ is invisible to node $u$, node $u$ also forwards the broadcast packet and ensures the coverage. Note that this method is conservative. If link $(v, w)$ is still available, making node $u$ a forward node causes extra redundancy. Using the dual neighbor set mechanism, the connectivity condition in Theorem 1 is relaxed without violating the link availability condition. It is sufficient that the physical network is connected with transmission range $r_{2}-\Delta^{\prime}$.

If the physical distance between two neighbors can be estimated based on the signal strength of a received message, the dual neighbor sets can be implemented via sending "Hello" messages with transmission range $r_{2}$. Each node classifies its neighbors into effective and advertised neighbors based on their estimated distances. When the signal strength is unavailable or inaccurate, each node will send two types of "Hello" messages. The first type of "Hello" messages, sent via transmission range $r_{2}$, are used to detect effective neighbors. The second, sent via transmission range $r_{1}$, are used to identify advertised neighbors.

\subsection{Analytical Study}

Based on Theorem 2, in order to guarantee that a neighbor (within $r_{1}$ ) at $t_{0}$ is an effective neighbor (within $r_{2}$ ) at a time $t_{1}=t_{0}+f, r_{1}$ must be smaller than $r_{2}-2 s f$ for a given maximal node speed $s$ and time period $f$. In this section, we show that the probability, $p$, that a node within $r_{1}$ at $t_{0}$ moves out of range $r_{2}$ at $t_{1}$ is reasonably small with a much larger $r_{1}$. We assume a mobility model similar to the random direction model [16], where each node is moving at a random speed in $[0, s]$ to a random direction in $[0,2 \pi]$. This is a simplified model for ease of probabilistic analysis. In addition, this model usually represents the worst case in terms of relative distance between two nodes in a given interval. 


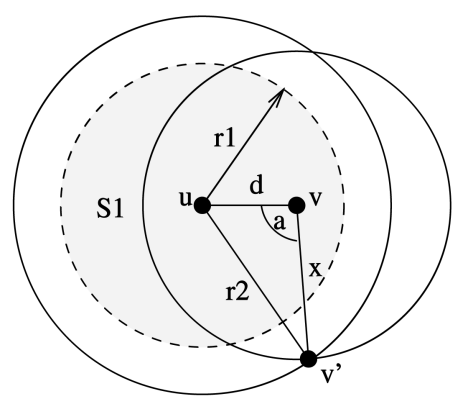

Fig. 10. Calculation of the probability that a neighbor within the "Hello" transmission range $\left(r_{1}\right)$ moves out of the normal transmission range $\left(r_{2}\right)$.

Consider two neighboring nodes $u$ and $v$ as shown in Fig. 10. Node $v$ is within $u^{\prime} \mathrm{s}$ "Hello" transmission range (the shadowed area) at time $t_{0}$ and moves to position $v^{\prime}$ at $t_{1}$. Assume that their distance at $t_{0}$ is $d$ and $v$ moves a distance of $x$ with respect to $u$ at $t_{1}$. The probability that $v$ moves out of the actual transmission range of $u$ is

$$
p(x, d)=\left\{\begin{array}{rll}
0 & : & x<r_{2}-d \\
1-\frac{\alpha}{\pi} & : & r_{2}-d \leq x \leq r_{2}+d \\
1 & : & x>r_{2}+d,
\end{array}\right.
$$

where $\alpha=\cos ^{-1}\left(\frac{x^{2}+d^{2}-r_{2}^{2}}{2 d x}\right)$ is the largest value of $\angle u v v^{\prime}$ that satisfies $d\left(u, v^{\prime}\right) \leq r_{2}$. The probability that any node within the "Hello" transmission range of $u$ moves out of its actual transmission range at $t_{1}$ is

$$
p(x)=\int_{0}^{r_{1}} \frac{2 \pi d}{S_{1}} p(x, d) \mathrm{d} d=\int_{0}^{r_{1}} \frac{2 d}{r_{1}^{2}} p(x, d) \mathrm{d} d,
$$

where $S_{1}=\pi r_{1}^{2}$ is the area within the "Hello" transmission range. The probability that a node with any constant relative speed $t$ with respect to $u$ moves out of the actual transmission range is

$$
p=\int_{0}^{2 s} f_{|\vec{V}|}(t) p(f t) \mathrm{d} t .
$$

Here, $\vec{V}=\vec{V}_{v}-\vec{V}_{u}$ is the random joint mobility vector between any two mobile nodes $u$ and $v$, where $\vec{V}_{u}\left(\vec{V}_{v}\right)$ is the random mobility vector of node $u(v)$. Note that (1) still holds, as the direction of $\vec{V}$ is also uniformly distributed in $[0,2 \pi]$ and is independent of the speed of $\vec{V},|\vec{V}|$. We know that $|\vec{V}|$ is between 0 and $2 s:|\vec{V}|=0$ when $\vec{V}_{u}=\vec{V}_{v},|\vec{V}|=2 s$ when $\vec{V}_{u}=-\vec{V}_{v}$, and $\left|\vec{V}_{u}\right|=\left|\vec{V}_{v}\right|=s$. However, its probability function, $f_{|\vec{V}|}(t)$, is unknown. McDonald and Znati [14] conducted a probabilistic analysis on the joint mobility of two nodes, but their analysis is based on the random walk mobility model [4], where the mobility vector of each node is the sum of several epochs and each epoch has different speed, direction, and duration. Li et al.'s analysis [13] is based on the same mobility model as ours, but their analysis is simplified by the implicit assumption that node $u$ is fixed and $|\vec{V}|$ is uniformly distributed in $[0, s]$. Here we calculate $f_{|\vec{V}|}(t)$ at a given $t$ as

$$
\begin{aligned}
f_{|\vec{V}|}(t) & \approx \frac{F_{|\vec{V}|}(t+\delta t)-F_{|\vec{V}|}(t)}{\delta t} \\
& =\frac{P(t \leq|\vec{V}| \leq t+\delta t)}{\delta t} \\
& =\oint_{(0,0)}^{(2 \pi, s)} \oint_{(0,0)}^{(2 \pi, s)} \frac{R\left(\vec{V}_{u}, \vec{V}_{v}, t, t+\delta t\right)}{(2 \pi s)^{2} \delta t} \mathrm{~d} \vec{V}_{u} \mathrm{~d} \vec{V}_{v},
\end{aligned}
$$

where $F_{|\vec{V}|}(t)$ is the distribution function, $\delta t$ is a small positive value, and

$$
R\left(\vec{V}_{u}, \vec{V}_{v}, a, b\right)=\left\{\begin{array}{lll}
1 & : & a \leq\left|\vec{V}_{v}-\vec{V}_{u}\right| \leq b \\
0 & : & \text { otherwise. }
\end{array}\right.
$$

Fig. 11a shows the distribution of $|\vec{V}|$ calculated from (4), when $s=1 \mathrm{~m} / \mathrm{s}$ and $\delta t=0.001 \mathrm{~m} / \mathrm{s}$. Note that the probability that $|\vec{V}|>1.5 \mathrm{~s}$ is small ( $\leq 5$ percent). Based on this distribution, we calculate the probability $p$ that any node within the "Hello" transmission range $\left(r_{1}=100,150,200\right.$, and 250) of $u$ moves out of its actual transmission range $\left(r_{2}=250 m\right)$ during a "Hello" interval $(f=1 s)$ when the maximal single node speed $s$ varies from 0 to $160 \mathrm{~m} / \mathrm{s}$. As shown in Fig. 11b, we can use an $r_{1}$ that is much larger than $r_{2}-2 s f$ and still expect a low probability that an effective neighbor moves out of the actual transmission range. For example, when $r_{1}=200 \mathrm{~m}$ and $s=80 \mathrm{~m} / \mathrm{s}$, the probability of losing an effective neighbor is less than 5 percent. Note that the corresponding $r_{1}$ that guarantees the availability of link $(u, v)$ at time $t_{1}$ is $r_{2}-2 s f=90 \mathrm{~m}$. When $r_{1}=100 \mathrm{~m}$ and $s=160 \mathrm{~m} / \mathrm{s}$, the probability of losing an effective neighbor is about the same. On the other hand, there is no $r_{1}$ that can

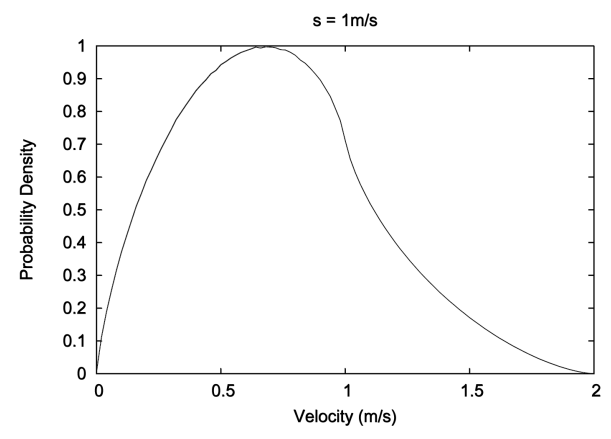

(a)

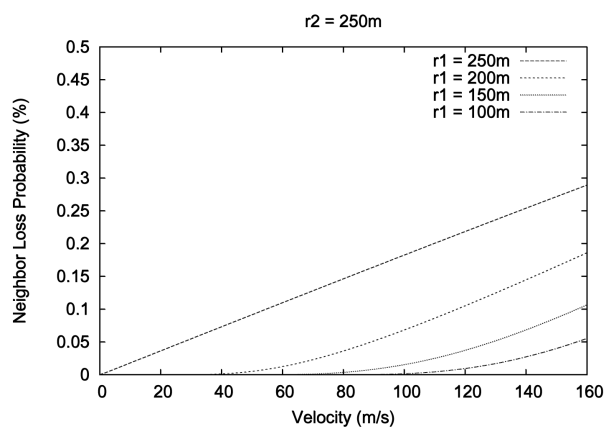

(b)

Fig. 11. (a) The probability function $f_{|\vec{V}|}(t)$ of the random joint mobility vector and (b) the probability that a neighbor within a given "Hello" transmission range $r_{1}$ moves out of the actual transmission range $r_{2}$. 
TABLE 1

Simulation Parameters

\begin{tabular}{l|l}
\hline Parameter & Value \\
\hline Network area & $900 \times 900 \mathrm{~m}^{2}$ \\
Actual transmission range & $250 \mathrm{~m}$ \\
"Hello" interval & $0.75-1.25 \mathrm{~s}$ \\
Simulation time & $100 \mathrm{~s}$ \\
Number of trials & 20 \\
Confidence level & $95 \%$ \\
\hline
\end{tabular}

guarantee the link availability, as $2 s f=320 m>r_{2}$.

\section{Simulation}

Simulations are conducted to evaluate the proposed method and explore appropriate "Hello" transmission ranges that achieve high delivery ratio with low broadcast redundancy under various mobility levels. We also evaluate the effectiveness of two implementation options that use dual neighbor sets to improve the delivery ratio under various environments.

\subsection{Simulation Environment}

The proposed mobility management method is simulated on $n s-2(1 b 7 a)$ [9] and its CMU wireless extension. We extend $\mathrm{Wu}$ and Dai's coverage condition by using two transmission ranges $r_{1}$ (for "Hello" messages) and $r_{2}$ (for actual transmission). When $r_{1}=r_{2}$, the new algorithm is equivalent to the original generic self-pruning protocol. We also simulate the dual neighbor sets enhancement for sparse networks. The consistency enforcement mechanisms (i.e., aggregated local views, delayed advertisement, and delayed removal) are not implemented.

The broadcast traffic rate is 10 packets per second with 64 bytes per packet. Each packet is issued from a randomly selected node. Since our purpose is to observe the behavior of self-pruning protocols under mobile environments, all simulations use an ideal MAC layer without contention or collision. If a node sends a packet, all neighbors within its transmission range will receive this packet after a short propagation delay. We assume that accurate location information is either unavailable or unable to predict the existence of wireless links due to the irregular variation of transmission range. It was shown in [7] that the contribution of a backoff delay to the protocol efficiency is trivial except for SBA. Therefore, our implementation of the proposed method does not use a backoff delay.

The mobility model used in the simulation is the random direction model [16]. In this model, each node moves towards a random direction at a random speed until it reaches the boundary of the area, where it selects new direction and speed and repeats the same process. Our mobility pattern generator is from [4], which has a parameter called average moving speed $\left(V_{\text {avg }}\right)$. For a given $V_{a v g}$, the speed of each node is randomly selected from the range $\left[0,2 V_{\text {avg }}\right]$. Note that the random direction model usually yields sparser networks and higher mobility than the commonly used random waypoint model [4]. Therefore, a reliable protocol in this simulation study is a reliable protocol under the random waypoint model, but not vice versa. Other simulation parameters are listed in Table 1.

\subsection{Simulation Results}

Fig. 12 shows simulation results in relatively dense networks (100 nodes), with buffer zone width (i.e., $r_{2}-r_{1}$ ) varying from $0 \mathrm{~m}$ to $100 \mathrm{~m}$. As expected, high delivery ratio ( $\geq 98$ percent) can be achieved with large buffer zone width $(100 \mathrm{~m})$ in highly mobile networks (with average speed $160 \mathrm{~m} / \mathrm{s})$. Note that this buffer zone width is much smaller than that required by Theorem 2, which should be at least $2 \times 2 V_{\text {avg }}=640 \mathrm{~m}$. This also confirms our prediction in Section 3.4 that high delivery ratio can be achieved using a small buffer zone. The only problem is the high broadcast cost ( $\geq 60$ percent forward nodes). If the network mobility level is known, we can select the buffer zone width based on the mobility level to balance the delivery ratio and redundancy. For example, at average speed $120 \mathrm{~m} / \mathrm{s}$, we can use a buffer zone width of $50 \mathrm{~m}$, which achieves 95 percent delivery ratio with 40 percent forward nodes. At average speed $40 \mathrm{~m} / \mathrm{s}$, a $10 \mathrm{~m}$ buffer zone achieves the same delivery ratio with only 30 percent forward nodes.

Fig. 13 shows the performance of the proposed method in relatively sparse networks ( 50 nodes). When a $0 m$ buffer zone is used, the delivery ratio drops rapidly as the average

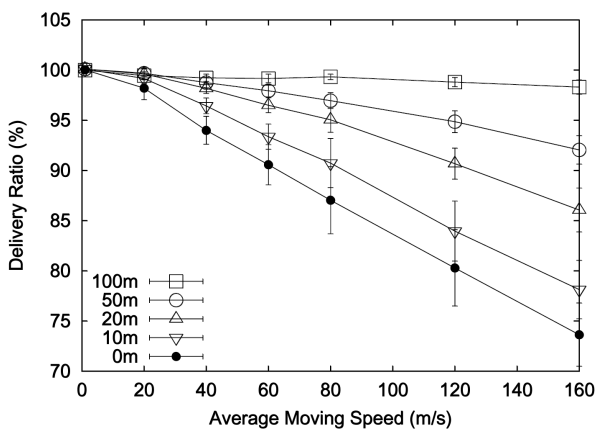

(a)

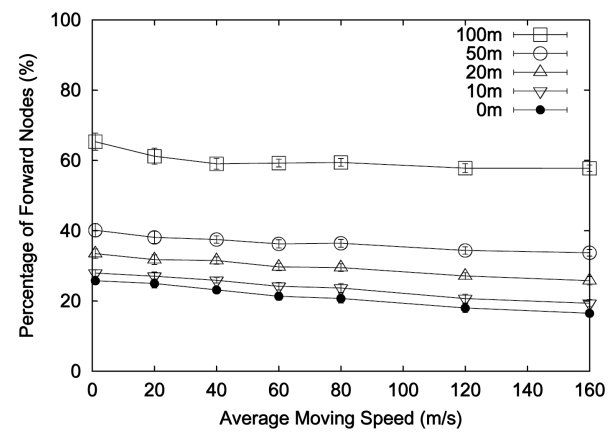

(b)

Fig 12. Performance of the original single neighbor set method in relatively dense (100 nodes) networks. (a) Delivery ratio. (b) Broadcast cost. 


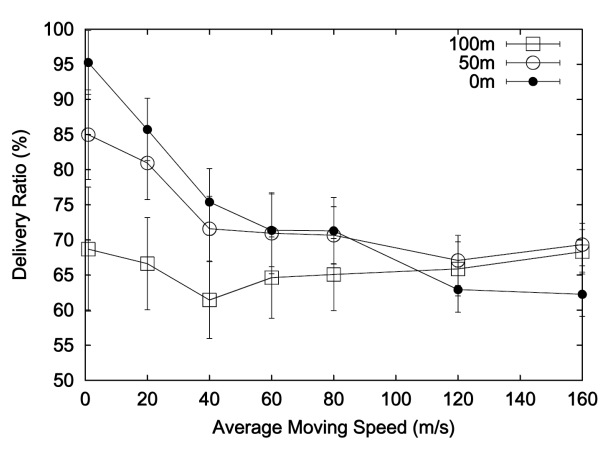

(a)

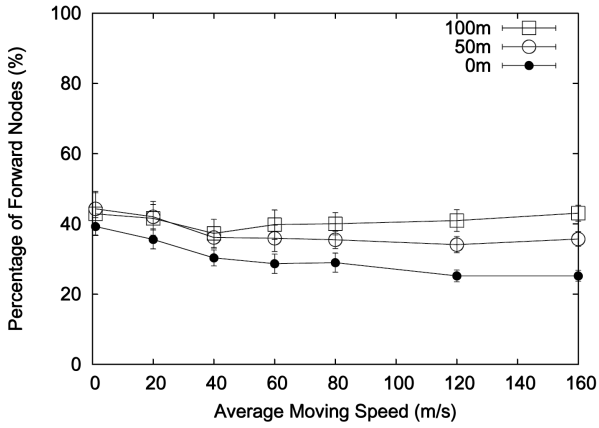

(b)

Fig. 13. Performance of the original single neighbor set method in relatively sparse (50 nodes) networks. (a) Delivery ratio. (b) Broadcast cost.

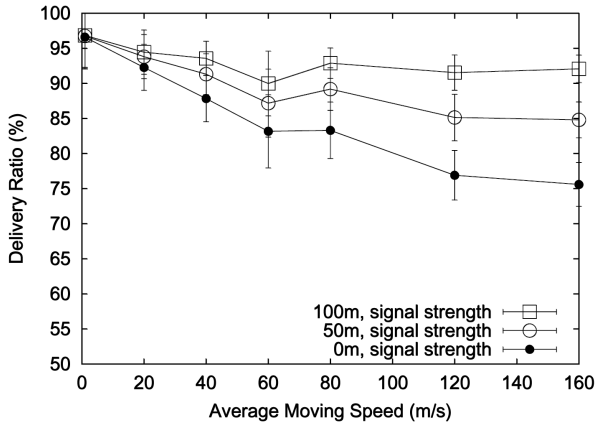

(a)

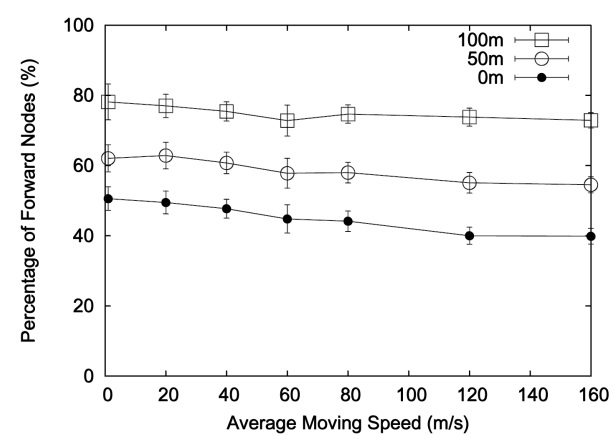

(b)

Fig. 14. Performance of the dual neighbor set enhancement in relatively sparse (50 nodes) networks. (a) Delivery ratio. (b) Broadcast cost.

speed increases. Using a larger buffer zone width $(\geq 50 \mathrm{~m})$ improves the delivery ratio under high mobility level, but performs poorly under low mobility level. The delivery ratio is low ( $<85$ percent), even with trivial mobility $(1 \mathrm{~m} / \mathrm{s})$. One reason for the low delivery ratio in sparse networks is the relatively low redundancy. The broadcast cost is low (40 percent) under different buffer zone widths. This is because the broadcast process fails in most cases, where only a few nodes receive and forward the packet.

Simulation results in [7] showed that all self-pruning protocols have lower delivery ratio in sparse networks than in dense networks under the same mobility level. Another reason is that when the network is not sufficiently dense, the connectivity requirement in Theorem 1 is not satisfied and, therefore, cannot guarantee the coverage. This problem can be solved with the dual neighbor set enhancement introduced in Section 3.3. Fig. 14 shows the performance of the enhanced scheme, where all neighbors within the actual transmission range $r_{2}$ are put into the effective neighbor set and only neighbors within transmission range $r_{1}$ are put into the advertised neighbor set. With this enhancement, high delivery ratio ( $\geq 90$ percent) can still be achieved under the highest mobility level. However, the corresponding broadcast cost is also higher (80 percent). Under low and median mobility $\left(V_{\text {avg }} \leq 40\right)$, a smaller buffer zone width $(50 \mathrm{~m})$ can be used to reduce the broadcast cost to 60 percent.

Overall, simulation results show that balance between delivery ratio and broadcast redundancy can be achieved by adjusting the buffer zone width based on the network mobility level. As predicted by our probabilistic analysis, for each mobility level, high delivery ratio can be achieved with a buffer zone much thinner than required by Theorem 2. The dual neighbor set enhancement is proved successful in relaxing the connectivity requirement in Theorem 1 and achieves high delivery ratio in sparse networks.

\section{Conclusions}

In this paper, we have proposed a mobility management method based on the use of two transmission ranges. Using this mechanism, we have also extended $\mathrm{Wu}$ and Dai's coverage condition to a dynamic environment where network topology is allowed to change, even during the broadcast process. In addition, connectivity, link availability, and consistency issues related to neighborhood information of different nodes have also been addressed. The proposed scheme can also be extended to provide mobility management for other activities, such as topology control in MANETs [25].

The constraint used on $r_{2}-r_{1}$ in this paper is conservative. Our probabilistic analysis suggests that high delivery ratio can still be achieved with a larger $r_{1}$. Simulation results show that the proposed method and the dual neighbor set enhancement achieve good balance between delivery ratio and broadcast redundancy by adjusting the value of $r_{1}$ based on the network mobility level. A future extension 
would be automatic buffer zone width adjustment that adapts to the neighborhood mobility level.

In $\mathrm{Wu}$ and Dai's coverage condition, node id is used to break a tie. We could also use the notion of relative mobility [1], defined as absolute relative speed averaged over time, for tie breaking. In general, a node with high relative mobility is more prone to unstable behavior than a node with less relative mobility and therefore should be pruned (from being a forward node) when possible. In this case, relative mobility is calculated locally through some form of approximation and distributed through piggybacking with regular "Hello" messages. Our future work also includes the adoption of vast results from the distributed system community with regards to global/local states and view consistency [18].

\section{ACKNOWLEDGMENTS}

This work was supported in part by US National Science Foundation grants ANI 0073736, CCR 0329741, CNS 0434533, CNS 0422762, and EIA 0130806.

\section{References}

[1] B. An and S. Papavassiliou, "A Mobility-Based Clustering Approach to Support Mobility Management and Multicast Routing in Mobile Ad-Hoc Wireless Networks," Int'l J. Network Management, vol. 11, no. 6, pp. 387-395, Nov./Dec. 2001.

[2] F. Bai, N. Sadagopan, and A. Helmy, "IMPORTANT: A Framework to Systematically Analyze the Impact of Mobility on Performance of Routing Protocols for Ad-Hoc Networks," Proc. IEEE Infocom, Mar./Apr. 2003.

[3] S. Basagni, I. Chlamtac, A. Farago, V.R. Syrotiuk, and R. Talebi, "Route Selection in Mobile Multimedia Ad Hoc Networks," Proc. IEEE MOMUC, Nov. 1999

[4] T. Camp, J. Boleng, and V. Davies, "A Survey of Mobility Models for Ad Hoc Network Research," Wireless Comm. E Mobile Computing (WCMC), special issue on mobile ad hoc networking: research, trends and applications, vol. 2, no. 5, pp. 483-502, 2002.

[5] B. Chen, K. Jamieson, H. Balakrishnan, and R. Morris, "Span: An Energy-Efficient Coordination Algorithm for Topology Maintenance in Ad Hoc Wireless Networks," Proc. MobiCom, pp. 85-96, July 2001.

[6] F. Dai and J. Wu, "An Extended Localized Algorithm for Connected Dominating Set Formation in Ad Hoc Wireless Networks," IEEE Trans. Parallel and Distributed Systems, vol. 15, no. 10, pp. 908-920, Oct. 2004.

[7] F. Dai and J. Wu, "Performance Comparison of Broadcast Protocols for Ad Hoc Networks Based on Self-Pruning," IEEE Trans. Parallel and Distributed Systems, vol. 15, no. 11, pp. 10271040, Nov. 2004.

[8] R. Dube, C. Rais, K. Wang, and S. Tripathi, "Signal Stability Based Adaptive Routing (SSA) for Ad Hoc Mobile Networks," IEEE Personal Comm., vol. 4, no. 1, pp. 36-45, Feb. 1997.

[9] K. Fall and K. Varadhan, "The ns Manual," The VINT Project, UCB, LBL, USC/ISI and Xerox PARC, http://www.isi.edu/ nsnam/ns/doc/, Apr. 2002.

[10] M. Grossglauser and D. Tse, "Mobility Increases the Capacity of Ad-Hoc Wireless Networks," Proc. IEEE Infocom, Apr. 2001.

[11] Z.J. Haas, J.Y. Halpern, and L. Li, "Gossip-Based Ad Hoc Routing," Proc. IEEE Infocom, June 2002.

[12] W.-I. Kim, D.-H. Kwon, and Y.-J. Suh, "A Reliable Route Selection Algorithm Using Global Positioning Systems in Mobile Ad-Hoc Networks," Proc. ICC, June 2001.

[13] N. Li, J.C. Hou, and L. Sha, "Design and Analysis of an MSTBased Topology Control Algorithm," Proc. IEEE Infocom, Mar./ Apr. 2003.

[14] A.B. McDonald and T.F. Znati, "A Mobility-Based Framework for Adaptive Clustering in Wireless Ad Hoc Networks," IEEE JSAC, Special Issue on Ad-Hoc Networks, Aug. 1999.

[15] W. Peng and X. Lu, "On the Reduction of Broadcast Redundancy in Mobile Ad Hoc Networks," Proc. MobiHoc, pp. 129-130, 2000.
[16] E. Royer, P. Melliar-Smith, and L. Moser, "An Analysis of the Optimum Node Density for Ad Hoc Mobile Networks," Proc. ICC, 2001.

[17] N. Sadagopan, F. Bai, B. Krishnamachari, and A. Helmy, "Paths: Analysis of Path Duration Statistics and their Impact on Reactive MANET Routing Protocols," Proc. MobiHoc, June 2003.

[18] M. Singhal and N.G. Shivaratri, Advanced Concepts in Operating Systems: Distributed, Database, and Multiprocessors Operating Systems. McGraw-Hill, Inc., 1994.

[19] I. Stojmenovic, M. Seddigh, and J. Zunic, "Dominating Sets and Neighbor Elimination Based Broadcasting Algorithms in Wireless Networks," IEEE Trans. Parallel and Distributed Systems, vol. 13, no. 1, pp. 14-25, Jan. 2002.

[20] W. Su, S.-J. Lee, and M. Gerla, "Mobility Prediction and Routing in Ad Hoc Wireless Networks," Int'l J. Network Management, vol. 11, no. 1, pp. 3-30, Jan.-Feb. 2001.

[21] J. Sucec and I. Marsic, "An Efficient Distributed Network-Wide Broadcast Algorithm for Mobile Ad Hoc Networks," CAIP Technical Report 248, Rutgers Univ., Sept. 2000.

[22] C.-K. Toh, "Associativity-Based Routing for Ad-Hoc Mobile Networks," IEEE Personal Comm., vol. , 4, no. 2, pp. 36-45, Mar. 1997.

[23] Y.-C. Tseng, S.-Y. Ni, and E.-Y. Shih, "Adaptive Approaches to Relieving Broadcast Storms in a Wireless Multihop Mobile Ad Hoc Network," IEEE Trans. Computers, vol. 52, no. 5, pp. 545-557, May 2003.

[24] J. Wu and F. Dai, "Broadcasting in Ad Hoc Networks Based on Self-Pruning," Proc. IEEE Infocom, Mar./Apr. 2003.

[25] J. Wu and F. Dai, "Mobility-Sensitive Topology Control in Mobile Ad Hoc Networks," Proc. IEEE Int'l Parallel and Distributed Processing Symp., May 2004.

[26] J. Wu and H. Li, "On Calculating Connected Dominating Set for

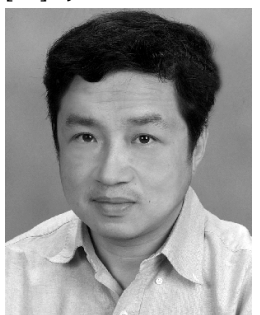
Efficient Routing in Ad Hoc Wireless Networks," Proc. DiaLM, pp. 7-14, 1999.

Jie $\mathbf{W u}$ is a professor in the Department of Computer Science, Florida Atlantic University. $\mathrm{He}$ has published more than 250 papers in various journals and conference proceedings and has served on many conference organization committees. He presently serves on the editorial board of IEEE Transactions on Parallel and Distributed Systems and was recently a co-guest-editor of IEEE Computer and the Journal of Parallel and Distributing Computing. He served as the program cochair for IEEE MASS 2004 and is the author of the text Distributed System Design, published by the CRC press. He was the recipient of the 1996-97 and 2001-2002 Researcher of the Year Award at Florida Atlantic University and served as an IEEE Computer Society Distinguished Visitor. His research interests are in the areas of mobile computing, routing protocols, fault-tolerant computing, and interconnection networks. He is a member of ACM and a senior

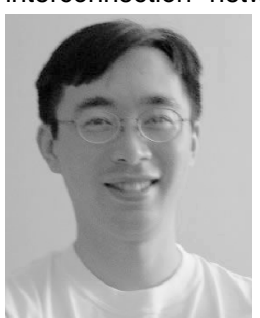
member of the IEEE.

Fei Dai received the BS and MS degrees in computer science from Nanjing University, Nanjing, China. He is currently a PhD candidate in the Department of Computer Science and Engineering, Florida Atlantic University. His recent research has focused on localized and self-organizing algorithms for wireless ad hoc and sensor networks. He has also conducted research study in the broad areas of networking, mobile computing, parallel and distributed computing, artificial intelligence, and software engineering. He has been a senior programmer in Greatwall Computer, China, for three years and a software architect and team leader in J\&A Securities, China, for four years. He is a student member of the IEEE.

For more information on this or any other computing topic, please visit our Digital Library at www.computer.org/publications/dlib. 